\title{
A dual role for Rac1 GTPases in the regulation of cell motility
}

\author{
Vedrana Filić ${ }^{1}$, Maja Marinović ${ }^{1}$, Jan Faix ${ }^{2, \star}$ and $\operatorname{Igor}_{\text {Weber }}{ }^{1, \star}$ \\ ${ }^{1}$ Ruder Bošković Institute, Division of Molecular Biology, Bijenička 54, HR-10000 Zagreb, Croatia \\ ${ }^{2}$ Hannover Medical School, Institute for Biophysical Chemistry, Carl-Neuberg-Str. 1, D-30625 Hannover, Germany \\ *Authors for correspondence (iweber@irb.hr; faix.jan@mh-hannover.de) \\ Accepted 28 July 2011 \\ Journal of Cell Science 125, 387-398 \\ (C) 2012. Published by The Company of Biologists Ltd \\ doi: 10.1242/jcs.089680
}

\section{Summary}

Rac proteins are the only canonical Rho family GTPases in Dictyostelium, where they act as key regulators of the actin cytoskeleton. To monitor the dynamics of activated Rac1 in Dictyostelium cells, a fluorescent probe was developed that specifically binds to the GTPbound form of Rac1. The probe is based on the GTPase-binding domain (GBD) from PAK1 kinase, and was selected on the basis of yeast two-hybrid, GST pull-down and fluorescence resonance energy transfer assays. The PAK1 GBD localizes to leading edges of migrating cells and to endocytotic cups. Similarly to its role in vertebrates, activated Rac1 therefore appears to control de novo actin polymerization at protruding regions of the Dictyostelium cell. Additionally, we found that the IQGAP-related protein DGAP1, which sequesters active Rac1 into a quaternary complex with actin-binding proteins cortexillin I and cortexillin II, localizes to the trailing regions of migrating cells. Notably, PAK1 GBD and DGAP1, which both bind to Rac1-GTP, display mutually exclusive localizations in cell migration, phagocytosis and cytokinesis, and opposite dynamics of recruitment to the cell cortex upon stimulation with chemoattractants. Moreover, cortical localization of the PAK1 GBD depends on the integrity of the actin cytoskeleton, whereas cortical localization of DGAP1 does not. Taken together, these results imply that Rac1 GTPases play a dual role in regulation of cell motility and polarity in Dictyostelium.

Key words: Rac1, Cell motility, Cell polarization, FRET, Actin cytoskeleton, Rho GTPases, Dictyostelium

\section{Introduction}

A major role of canonical small GTPases from the Rho subfamily in regulation of the actin cytoskeleton was first demonstrated in 1995 (Nobes and Hall, 1995). In agreement with results of these seminal studies, a general notion prevails that Rac and $\mathrm{Cdc} 42$ are primarily engaged in the initiation of protrusive structures such as lamellipodia and filopodia at the front of motile cells, whereas Rho predominantly regulates contractile structures in the back. However, evidence obtained in various cell types indicates that the spatial distribution of these proteins can be more complex (Heasman et al., 2010; Machacek et al., 2009; Pestonjamasp et al., 2006).

In mammalian cells, actin polymerization appears to be induced by two major routes: GTP-bound Rac1 and $\mathrm{Cdc} 42$ trigger Scar/WAVE- and WASP-mediated activation of the Arp2/ 3 complex (Iden and Collard, 2008), whereas activated Rho proteins release autoinhibition of Diaphanous-related formins (Faix and Grosse, 2006). In addition to their regulatory role in cell protrusion, $\mathrm{Rac} 1$ and $\mathrm{Cdc} 42$ have been implicated in numerous other processes involving actin polymerization, such as endocytosis, vesicular transport, secretion, cell polarization and cytokinesis (Heasman and Ridley, 2008). Small GTPases are activated by guanine nucleotide exchange factors (GEFs) and deactivated by GTPase-activating proteins (GAPs), and each of these two groups of regulating proteins is represented by more than 70 members encoded in the human genome (Rossman et al., 2005; Tcherkezian and Lamarche-vane, 2007). Deactivation of small GTPases is also attributed to a less numerous group of guanine nucleotide dissociation inhibitors (GDIs) that sequester the GTPases in a GDP-bound state in the cytoplasm, rendering them inaccessible to activators at the plasma membrane (DerMardirossian and Bokoch, 2005).

Highly motile cells of Dictyostelium discoideum represent a rewarding model in which regulation of the actin cytoskeleton dynamics is investigated (Noegel and Schleicher, 2000). Dictyostelium genome analysis revealed 18 Rho GTPases, among them several members of the Rac subfamily, whereas no typical Rho and Cdc42 subfamily members were identified (Vlahou and Rivero, 2006). Several Rac proteins have been investigated, indicating that their ubiquitous role in regulation of the actin cytoskeleton is conserved in Dictyostelium, but more detailed analyses of their dynamics and activity in living cells are still lacking (Rivero and Somesh, 2002). Such information is of major interest to complement data on related signalling pathways from other cell types and organisms (Heasman and Ridley, 2008; Wang and Zheng, 2007). Moreover, regulation of a rich repertoire of actin-driven activities in Dictyostelium, strongly resembling the dynamics of highly motile mammalian cells, has to be mapped to a specific set of canonical Rho GTPase proteins. Consequently, important insights into general principles of regulation of the motile machinery in eukaryotic cells and its diversity, robustness and evolution could be gained.

To study the dynamics of Rac proteins in Dictyostelium, we designed a fluorescent probe specific for the activated form of Rac1 GTPases and monitored its localization in migrating cells. Our probe displayed a clear temporal and spatial coincidence 
with structures that are propelled by actin polymerization, such as protruding leading edges and expanding endocytotic cups. Comparison with another Rac1-GTP-binding protein, IQGAPrelated DGAP1, which localizes to retracting trailing regions of migrating cells, suggests that activated Rac1 plays at least two different roles in the regulation of the actin cytoskeleton.

\section{Results}

\section{Rac1A is distributed homogenously in the cell cortex}

When expressed as GFP fusion proteins in Dictyostelium, wildtype forms of highly similar GTPases Rac1A, Rac1B and Rac1C show a slight enrichment in the cell cortex compared with a prominent cytoplasmic background (Dumontier et al., 2000). We decided to take a closer look at Rac1A localization in processes that involve remodelling of the actin cytoskeleton. GFP-tagged Rac1A is homogenously distributed throughout the cell cortex during migration of vegetative Dictyostelium cells, showing no preference for either protruding or retracting regions (Fig. 1A). GFP-Rac1A is also constantly present in the perinuclear region and in endocytotic cups during macropinocytosis and phagocytosis (Fig. 1A,C). Its cortical localization is, however, less prominent during cytokinesis, and barely discernible during chemotaxis of aggregation competent cells (Fig. 1B,D).

Distribution and dynamics of the GFP-Rac1A fusion protein in living cells presumably mirrors the distribution and dynamics of endogenous Rac1A. However, information conveyed in this way bears two important limitations. First, the total population of Rac1A molecules in the cell represents a mixture of an active, GTP-bound form and an inactive, GDP-bound form. Therefore, distribution and dynamics of GFP-Rac1A per se does not provide spatial and temporal information about the intracellular activity of the GTPase. Additionally, a more than tenfold overexpression level of the fusion protein compared with endogenous Rac1A probably leads to a high degree of its retention in the cytoplasm and, consequentially, to an exceedingly strong cytoplasmatic fluorescent background (Dumontier et al., 2000).

\section{PAK1 GBD interacts with the active form of Rac1A in vitro} To obtain information about the intracellular distribution of Rac1 activity, we decided to generate a fluorescent probe based on a domain that interacts specifically with the active, GTP-bound Rac1A. As a first step in the selection of an appropriate GTPasebinding domain, we performed a yeast two-hybrid (Y2H) screen using the following four candidate domains as baits: a full-length and a truncated form of the Rac1A-interacting protein DGAP1 (Faix et al., 1998), the GTPase-binding domain of Dictyostelium DPAKa kinase (Müller-Taubenberger et al., 2002) and the regulatory, GTPase-binding domain of rat PAK1 kinase (Buchwald et al., 2001). In addition to constitutively active forms of 11 Dictyostelium Rac proteins, we included the constitutively inactive forms of Rac1A, Rac1C and RacC as preys in the Y2H screen. The PAK1 GBD (amino acid residues 57-200) interacted with active forms of Rac1A, Rac1C and RacC, but not with their inactive forms, under both lowstringency and high-stringency conditions (Fig. 2A). We therefore chose this probe to further study its interaction with active Rac1A.

Two variants of GST-binding pull-down assay were used to verify that PAK1 GBD interacts with the active form of Rac1A, but not with its inactive form. First, a GST-[PAK1(GBD) $]_{2}$ construct was expressed in $E$. coli and used to affinity-purify GFP-fusion proteins from lysates of cells that express the following constructs: GFP-Rac1A(WT), GFP-Rac1A(V12), GFP-Rac1A(N17) and GFP-RacC(WT). Immunoprecipitation of bound proteins with antibodies against GFP showed that the PAK1 GBD binds to constitutively active mutant Rac1A(V12), but not to constitutively inactive mutant Rac1A(N17) (Fig. 2B). The PAK1 GBD also interacts with the wild-type forms of Rac1A
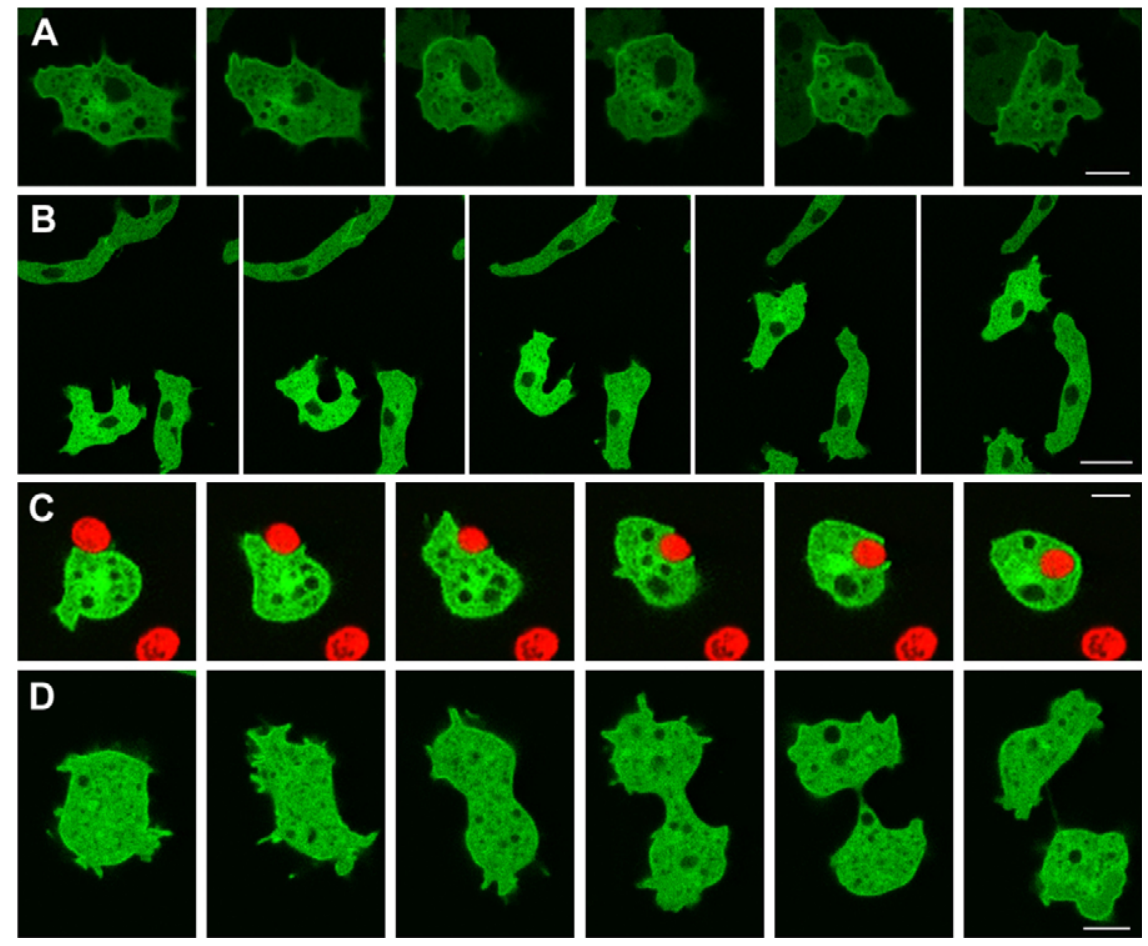
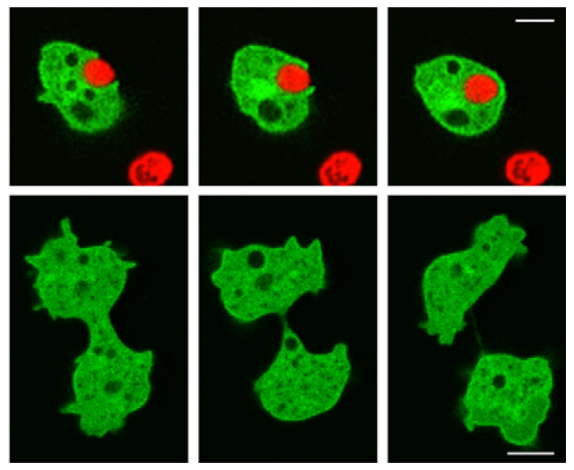

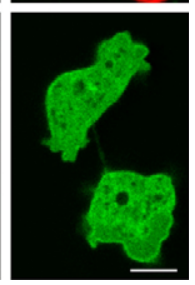

Fig. 1. Localization of GFP-Rac1A(WT) protein in wild-type D. discoideum cells. (A) Random movement. (B) Directed migration of aggregation competent cells. (C) phagocytosis. (D) Cytokinesis. During chemotaxis assay (B), the source of chemoattractant was located $30 \mu \mathrm{m}$ above the upper right corner of the displayed frame. Phagocytosis was induced using fluorescent, TRITC-labelled yeast particles. Scale bars: $5 \mu \mathrm{m}$ (A,C,D); $10 \mu \mathrm{m}$ (B). Time spans: 190 seconds (A); 130 seconds (B); 92 seconds (C); 255 seconds (D). 
A

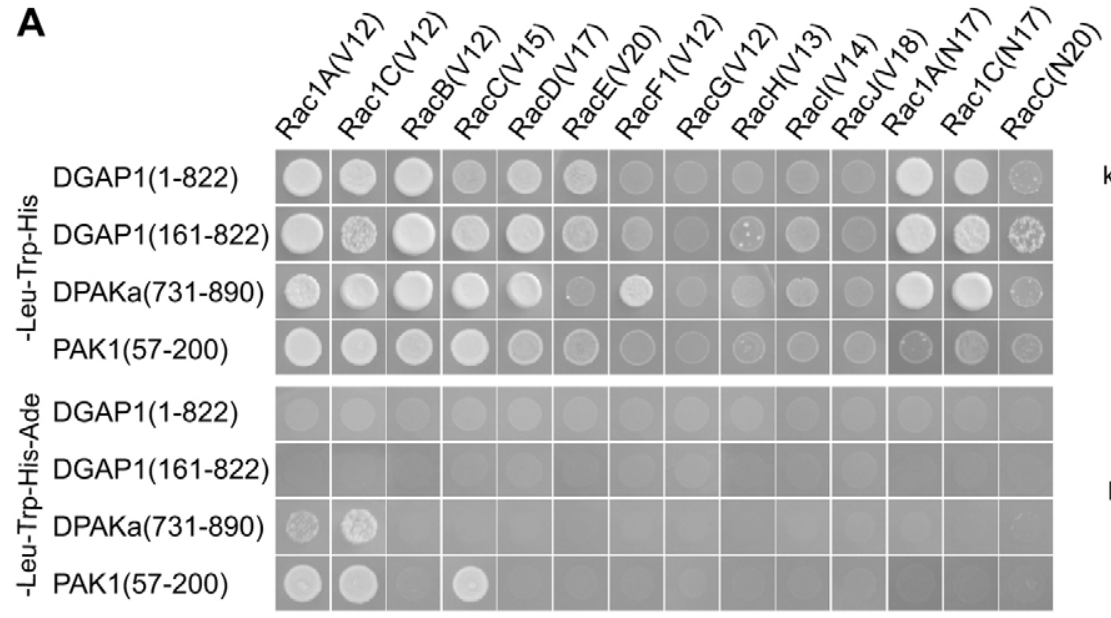

B

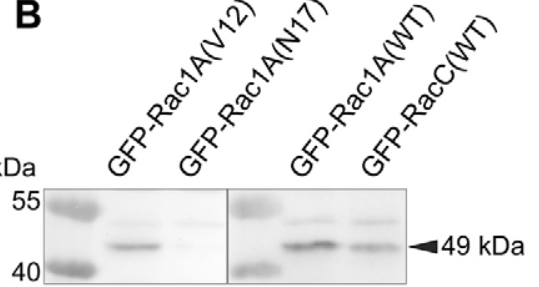

C GST-Rac1A

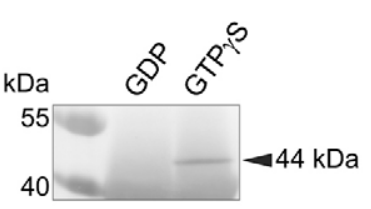

Fig. 2. The regulatory domain of rat PAK1 kinase specifically interacts with active forms of Dictyostelium Rac1 and RacC GTPases. (A) Yeast two-hybrid assay was used to test interactions between full-length and truncated variants of Dictyostelium DGAP1, the GBD of Dictyostelium DPAKa kinase, and the regulatory domain of rat PAK1 kinase as baits, with constitutively active forms of 11 Dictyostelium Rac proteins and constitutively inactive forms of three of these proteins as preys. Only PAK1 GBD (aa 57-200) interacts with active forms of Rac1A, Rac1C and RacC, but not with their inactive forms, under both low stringency (top panel) and high stringency (bottom panel) conditions. (B) GST-binding assay shows interactions of PAK1 GBD with wild-type forms of Rac1A and RacC, and with constitutively active form of Rac1A, but not with constitutively inactive Rac1A. GST-[PAK1(GBD) $]_{2}$ recombinant protein was bound to glutathione-Sepharose and used to affinity purify GFP fusion proteins from whole-cell lysates, as indicated. After elution proteins were immunoprecipitated with anti-GFP antibodies. (C) GST-Rac1A was bound to glutathione-Sepharose and charged with either GDP or GTP $\gamma$ S as indicated. Using whole-cell lysate, GBD probe was immunoprecipitated with anti-PAK1 GBD antibodies exclusively from the column containing GTP $\gamma$ S-loaded GST-Rac1A.

and RacC. In another GST pull-down assay, recombinant GSTRac1A was coupled to gluthathione-Sepharose beads and loaded with either GDP or the non-hydrolysible GTP analogue GTP $\gamma$ S. Proteins from lysate of cells expressing PAK1(GBD)DYFP fusion protein that bound to functionalized beads were immunoprecipitated with antibodies against PAK1 GBD only from the column containing GTP $\gamma$ S-loaded GST-Rac1A (Fig. 2C).

\section{FRET probes demonstrate an interaction between the PAK1 GBD and the active form of Rac1A in living cells}

Fluorescence (or Förster) resonance energy transfer (FRET) is an established method for probing binary interactions between fluorescently labelled molecules in living cells (Zhang et al., 2002). In particular, it has been successfully applied to map the sites of intracellular activity of small Rho GTPases in leukocytes, fibroblasts and other cell types (Pertz and Hahn, 2004). We adapted this approach to examine the interaction between PAK1 GBD and Rac1A in living Dictyostelium cells. The following unimolecular FRET probes were constructed and expressed in wild-type cells: Rac1A(WT) $)_{\text {FRET }}$, Rac1A(V12) $)_{\text {FRET }}$ and

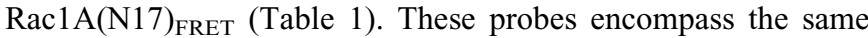

PAK1 GBD domain as used in yeast two-hybrid and pull-down assays. A schematic presentation of the Rac1A(WT) $)_{\text {FRET }}$ probe is shown in Fig. 3A. All probes contain a C-terminal CAAX motif to allow appropriate anchorage of the probe into the plasma membrane analogous to endogenous Rac1 proteins.

To determine the extent of the FRET effect, we measured sensitised emission of the acceptor (mRFPmars) upon excitation of the donor (DYFP). The cells expressing a FRET construct were mixed with cells expressing either only the donor or the acceptor. The recorded images always included at least one cell of each species (Fig. 3C), and corrections for bleed-through and cross-excitation were performed separately for each analyzed image. Calculations of FRET efficiency were performed on a pixel-by-pixel basis (Fig. 3D). Two probes, Rac1A(WT) FRET $_{\text {and }}$ Rac1A(V12) $)_{\text {FRET }}$, were localized in the cell cortex and in the perinuclear region, resembling to a large extent the localization of GFP-Rac1A, but with a lower background level in the cytoplasm (Fig. 3C). The third probe, Rac1A(N17) $)_{\text {FRET }}$, was localized in the cytoplasm with no significant cortical enrichment (data not shown). For each measured cell, average FRET efficiency $E$ in the cell cortex was calculated (see the Materials and Methods).

Table 1. Nomenclature of fluorescent probes

\begin{tabular}{lll}
\hline Composition of construct & \multicolumn{1}{c}{ Description of probe } & Abbreviation \\
\hline DYFP-PAK1(GBD)-Rac1A(WT)-mRFPmars & FRET probe for wild-type Rac1A & Rac1A(WT) $)_{\text {FRET }}$ \\
DYFP-PAK1(GBD)-Rac1A(V12)-mRFPmars & FRET probe for constitutively activated Rac1A & Rac1A(V12) \\
DYFP-PAK1(GBD)-Rac1A(N17)-mRFPmars & FRET probe for constitutively inactivated Rac1A & Rac1A(N17) \\
DYFP-PAK1(GBD)-mRFPmars & FRET probe for wild-type RacC & RacC(WT) \\
DYFP-PAK1_GBD-mRFPmars & FRET probe for endogenous activated Rac1 (negative control) & Neg \\
DYFP-mRFPmars & FRET probe for constitutive FRET (positive control) & PoS \\
PAK1(GBDET -DYFP & Probe for activated Rac1 in the cell front & GBD probe \\
mRFPmars-DGAP1 & Probe for the quaternary complex in the cell back that includes Rac1 & DGAP1 probe \\
& (its assembly depends on binding of activated Rac1) & \\
\hline
\end{tabular}


A

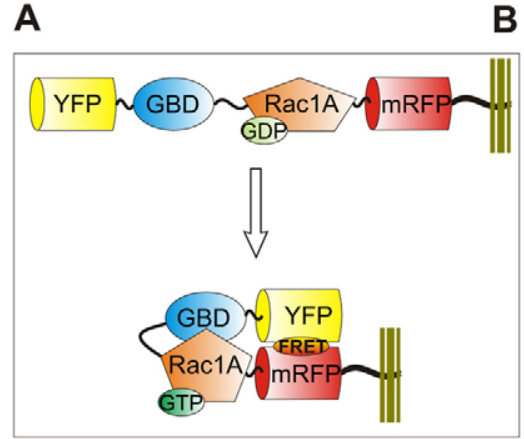

C

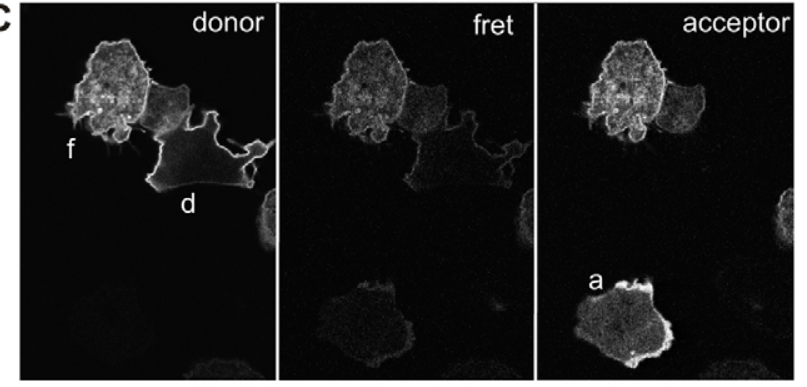

D
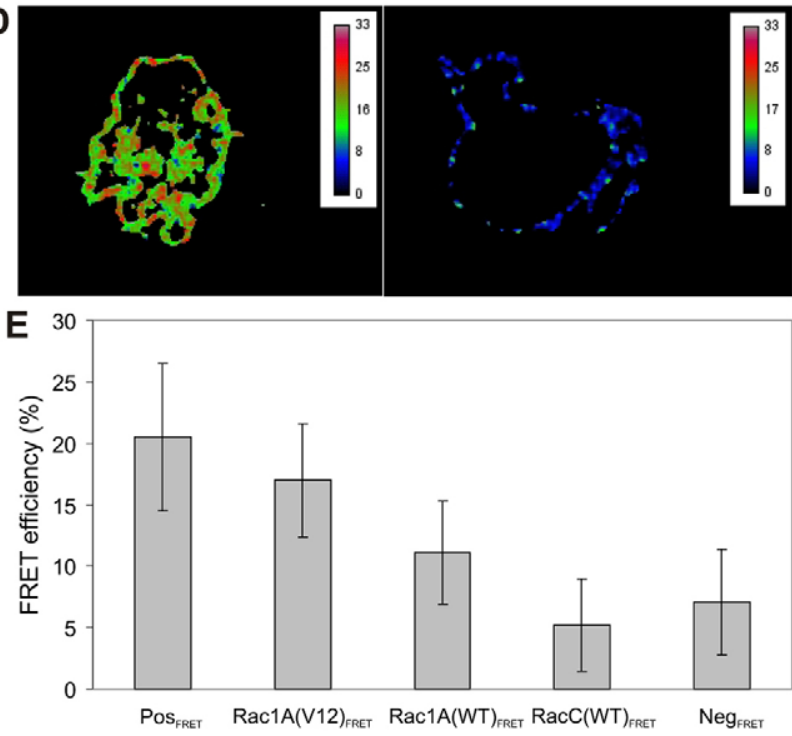

Fig. 3. Intramolecular constructs used for measurements of FRET efficiency. (A) Quadruple construct Rac1A(WT) $)_{\text {FRET }}$ reports FRET upon interaction between Rac1A-GTP and PAK1 GBD. In Rac1A(V12) Rac1A(N17) $)_{\text {FRET }}$ constructs, wild-type Rac1A is replaced by the constitutively active and constitutively inactive mutants of Rac1A, respectively. (B) Triple

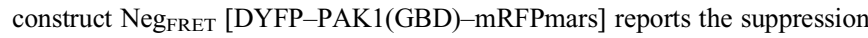
of constitutive FRET in the cell cortex upon interaction between endogenous Rac1A-GTP and PAK1 GBD. (C) An example of raw data images where three

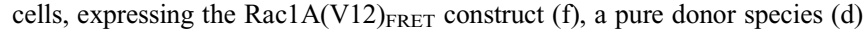
and a pure acceptor species (a) were sequentially recorded in donor, fret and acceptor channels. (D) Colour-coded maps of the FRET efficiency as

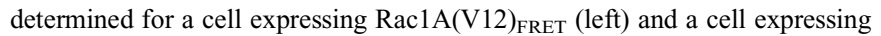
$\mathrm{Neg}_{\text {FRET }}$ (right). (E) Apparent FRET efficiencies measured for the five designated constructs (mean \pm s.d.).

Apparent FRET efficiencies of the positive control, $E\left(\operatorname{Pos}_{\mathrm{FRET}}\right)=20.5 \pm 6.0 \quad(n=12), \quad$ and $\quad E\left[\operatorname{Rac} 1 \mathrm{~A}(\mathrm{~V} 12)_{\mathrm{FRET}}\right]=$ $17.0 \pm 4.6(n=10)$, are statistically indistinguishable $(P>0.15)$, which suggests an interaction between PAK1 GBD and activated
Rac1A in vivo (Fig. 3E). We next compared FRET efficiency of the Rac1 $\mathrm{A}(\mathrm{V} 12)_{\mathrm{FRET}}$ construct with a tripartite construct DYFPPAK1(GBD)-mRFPmars that served as a negative control, $\mathrm{Neg}_{\text {FRET }}$ (Fig. 3B). Although this construct is designed to enable constitutive FRET, interaction of the PAK1 GBD with endogenous Rac1A-GTP precludes close contact between DYFP and mRFPmars, and thereby diminishes FRET (Itoh et al., 2002). $E\left[\operatorname{Rac} 1 \mathrm{~A}(\mathrm{WT})_{\mathrm{FRET}}\right]=11.1 \pm 4.2(n=10)$ was significantly higher than $E\left(\mathrm{Neg}_{\text {FRET }}\right)=7.1 \pm 4.3(n=13 ; P<0.05)$, indicating a GEF activity in the cell cortex that activates both endogenous and ectopically expressed Rac1A (Fig. 3E). Owing to a low signal-tonoise ratio, it was not possible to reliably measure FRET efficiency of the Rac1A(N17) $)_{\text {FRET }}$ probe.

\section{GBD probe is transiently recruited to the cell cortex upon} stimulation with folic acid and CAMP

We next expressed PAK1(GBD)-DYFP (abbreviated hereafter to 'GBD probe', see Table 1) in wild-type cells and in cells that overexpress mRFPmars-Rac1A(WT) fusion protein and performed a series of uniform stimulation experiments using folic acid and cAMP as chemoattractants. The primary goal was to test whether activation of Rac1 GTPases is triggered by these stimuli, which would indicate that Rac1 is participating in signalling pathways downstream of heterotrimeric $G$ proteins in Dictyostelium (Van Haastert and Devreotes, 2004). Additionally, an enhanced response to chemoattractants in cells that overexpress Rac1A would be an independent verification of an interaction between GBD probe and the active form of Rac1A in living cells, shown by FRET probes. Stimulation of vegetative cells by a pulse of folic acid induced a transient recruitment of GBD probe to the cell cortex lasting approximately 12 seconds (Fig. 4A,B). Cortical enrichment of the probe was stronger in cells overexpressing mRFPmars-Rac1A(WT) than in wild-type cells, whereas pulse-induced enrichment of mRFPmarsRac1A(WT) was barely visible. Similar results were obtained when early stage aggregation-competent cells were stimulated by a pulse of cAMP (Fig. 4C,D), albeit with a lower cortical contrast. An overall lower ratio between intensities of cortical versus cytoplasmatic GBD probe in aggregation competent cells is due to a gradually diminishing level of the probe in the course of starvation (western analysis, data not shown).

Results of the uniform stimulation experiments corroborated that the GBD probe interacts with activated Rac1 in living cells, and that Rac1 activation can be triggered by G-protein-mediated signal transduction pathways. Kinetics of Rac1 activation in the cortex, as visualized by cortical enrichment of the GBD probe, is comparable to the kinetics of cortical enrichment of Ras and phosphatidylinositol-3,4,5-trisphosphate probes upon stimulation with folic acid and cAMP (Sasaki et al., 2007).

\section{GBD probe is transiently recruited to leading edges, dorsal ruffles and endocytotic cups and localizes to sites of active actin assembly}

On the basis of a specific interaction between PAK1 GBD and activated Rac1A that was demonstrated in vitro and in vivo, we decided to use the GBD probe as a reporter of active Rac1 in living cells. In contrast to Rac1A(WT) $)_{\text {FRET }}$, which was localized to the cortical layer of motile cells uniformly and continuously, localization of GBD probe to the cell cortex was spatially confined and transient. In migrating vegetative cells, GBD probe 


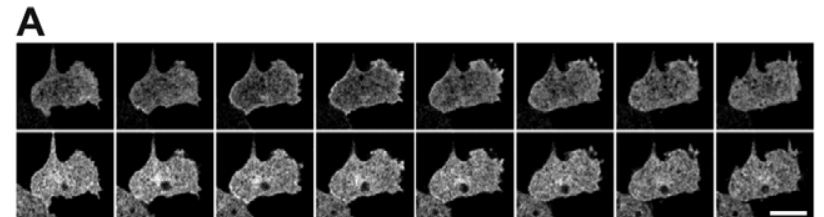

B
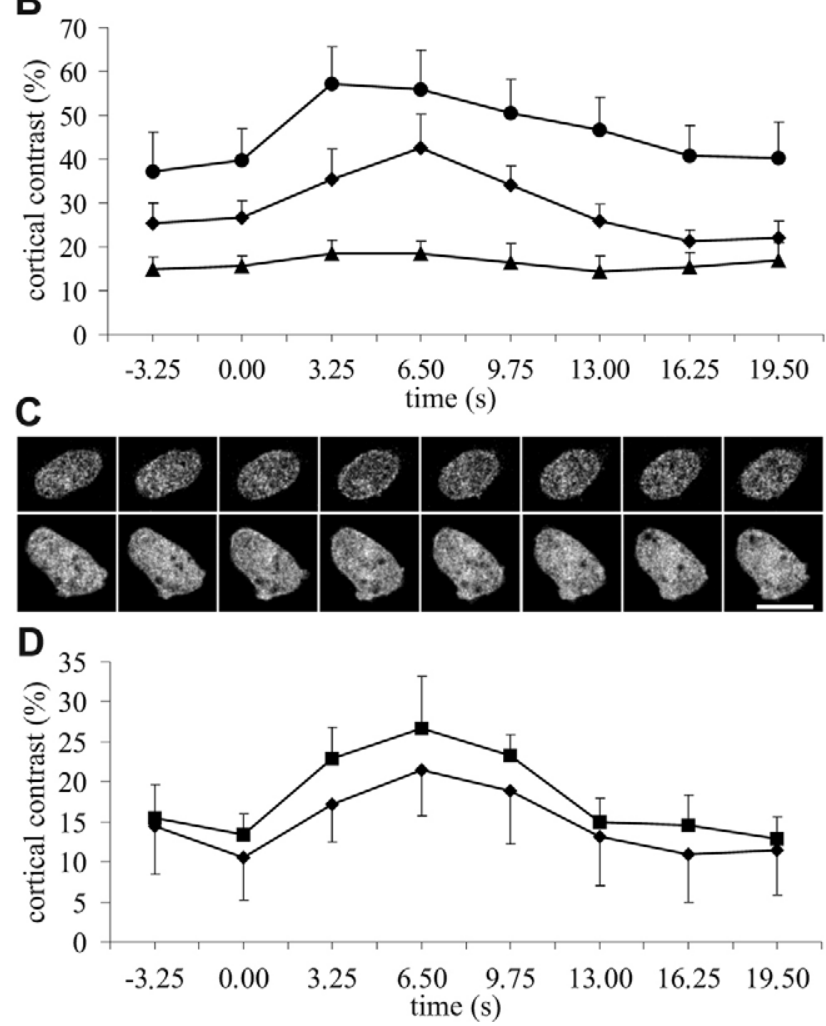

Fig. 4. Translocation of fluorescent probes to the cell cortex upon pulse stimulation by folic acid and cAMP. (A) Typical response of a cell that coexpresses the GBD probe (top row) and mRFPmars-Rac1A (bottom row) upon stimulation with folic acid. (B) Time courses of the cortical enrichment of GBD probe in wild-type cells $(\bullet)$, GBD probe in Rac1A-overexpressing cells (•), and mRFPmars-Rac1A in Rac1A-overexpressing cells (४) upon application of $50 \mu \mathrm{M}$ folic acid. (C) Typical dynamics of the cortical enrichment of GBD probe in a Rac1A-overexpressing cell (top row) and in a wild-type cell (bottom row) upon stimulation with cAMP. (D) Time courses of the cortical enrichment of GBD probe in wild-type cells $(\downarrow)$, and in Rac1A-overexpressing cells ( $\mathbf{a})$ upon application of $30 \mu \mathrm{M}$ cAMP. Cortical contrast designates average enrichment of the fluorescent signal in the cell cortex as compared with the cytoplasm (B,D; see the Materials and Methods) Shown results are averaged over six cells from at least three independent experiments (mean \pm s.e.m.). Scale bars: $10 \mu \mathrm{m}$. Times in seconds relate to the time-point of application of the pulse (B,D). Acquisition times of images in $\mathrm{A}$ and $\mathrm{C}$ correspond to measurement points in $\mathrm{B}$ and $\mathrm{D}$.

was enriched in the cortical protruding regions and dorsal ruffles of polarized cells (Fig. 5A). During chemotaxis of aggregationcompetent cells, however, cortical or polarized enrichment of the probe could no longer be detected (Fig. 5B). GBD probe also localized to nascent macropinosomes (Fig. 5A, frames 4 and 5; Fig. 5C, frame 4) and phagosomes (Fig. 5C), but was not enriched in the cortex during cytokinesis, either at the poles or in the cleavage furrow (Fig. 5D).

Because GBD probe transiently accumulated at sites of active actin assembly in migration and endocytosis, we correlated spatial distribution of active Racl detected by our probe with localization of polymerized actin by co-expressing GBD probe with mRFPmars-LimE $\Delta$ coil, an established probe of filamentous actin in Dictyostelium cells (Gerisch et al., 2004). The two markers colocalized in leading edges and endocytotic cups, indicating a close association between Racl activity and treadmilling actin filaments in these structures (Fig. 5E; supplementary material Movie 1). Kinetics of the cortical recruitment of the two probes upon stimulation by folic acid also indicated temporal coincidence of Rac1 activation and actin polymerization in the cell cortex within the limits of our time resolution (Fig. 5F,G).

\section{GBD probe specifically interacts with Rac1, and not RacC, in living cells}

Results of the yeast two-hybrid and GST pull-down assays indicated that, besides its interaction with activated Rac1 GTPases, PAK1 GBD is also capable of interacting with activated RacC in vitro (Fig. 2). To determine whether this interaction occurs in the cortex of living cells, we performed two control experiments. First, we expressed a GFP-RacC(WT) fusion protein in Dictyostelium and monitored its localization in motile cells. GFP-RacC(WT) was accumulated in cytoplasmatic granules but was not enriched in the cell cortex (supplementary material Fig. S1). From the localization data, it therefore appears that Rac1 and RacC localize to non-overlapping compartments within the cell (cf. Fig. 1 and supplementary material Fig. S1). Our GBD probe also never displayed localization that resembled that of GFP-RacC(WT), which suggests that PAK1 GBD and RacC do not interact in vivo.

To strengthen this conclusion, we also constructed a unimolecular FRET probe where Rac1A in the Rac1A(WT) $)_{\text {FRET }}$ construct was replaced with RacC, RacC(WT) $)_{\text {FRET. }}$ FRET experiments performed with $\operatorname{RacC}(\mathrm{WT})_{\text {FRET }}$ resulted in a low FRET efficiency, $E\left[\operatorname{RacC}(\mathrm{WT})_{\mathrm{FRET}}\right]=5.2 \pm 3.8(n=10)$, which was significantly lower than the value for Rac1A(WT) $)_{\text {FRET }}(P<0.01)$ (Fig. 3E). Taken together, these results indicate that RacC is generally not present in the cell cortex, and when its cortical localization is enforced, it still does not interact with the PAK1 GBD. We therefore conclude that the GBD probe specifically labels cortical sites of Rac1 activation.

\section{GBD probe and DGAP1 both interact with activated Rac1, but show distinct localization in migrating cells}

DGAP1 is an IQGAP-related protein from Dictyostelium that preferentially binds the activated form of Racl (Dumontier et al., 2000), and is part of a quaternary complex also containing a heterodimer of actin-bundling proteins cortexillin I and cortexillin II (Faix et al., 2001). Charging of Rac1 with GTP is mandatory for the assembly of this quaternary complex in vitro and in vivo. This Rac1-containing complex plays a crucial role in driving cytokinesis in Dictyostelium, and accumulates in the cleavage furrow of dividing cells. We first examined localization of a component of the complex, cortexillin I, in migrating cells. We used GFP-labelled C-terminal region of cortexillin I (residues 352-444), which is sufficient for targeting cortexillin I to the cell cortex, for the rescue of cytokinesis (Weber et al., 1999a), and for the interaction with DGAP1 (Faix et al., 2001). GFP-cortexillinI(352-444) localized to the cortex of unipolar and multipolar cells, but was excluded from protruding regions (Fig. 6A), which is the opposite to the localization of the GBD probe. 

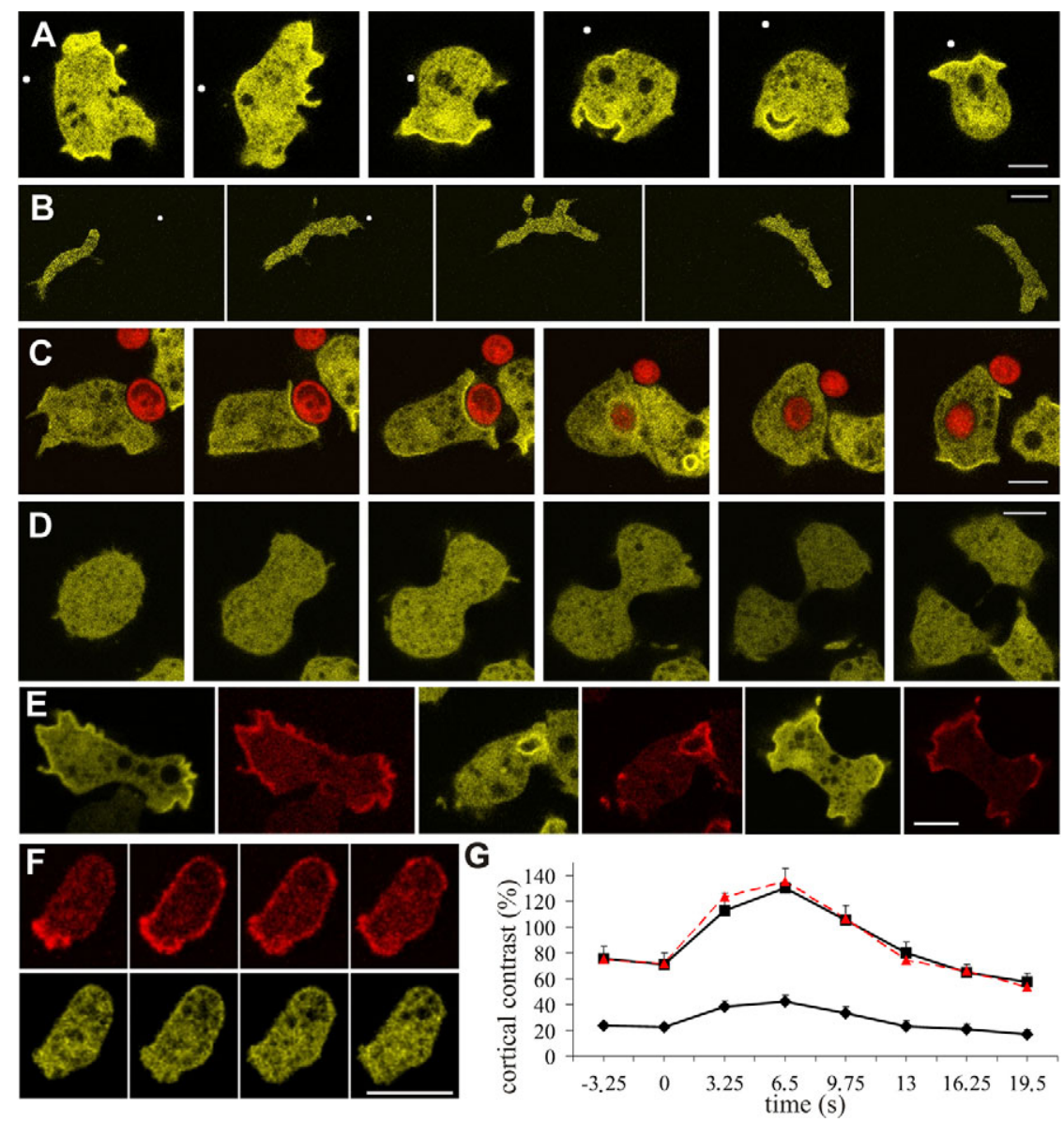

Fig. 5. Localization of GBD probe in wild-type

Dictyostelium cells. (A) Random movement. (B) Directed movement of aggregation competent cells.

(C) Phagocytosis. (D) Cytokinesis. (A) White dots designate a fixed reference point on the substratum. Note that the cell moved significantly downward between the third and the fourth frame. (B) White dots mark the position of the micropipette tip, i.e. the source of chemoattractant, in the first two frames. Afterwards, the micropipette was relocated $20 \mu \mathrm{m}$ underneath the lower left corner of the displayed frame. (C) Phagocytosis was induced using fluorescent, TRITC-labelled yeast particles. (E) Colocalization of co-expressed GBD probe (yellow) and mRFPmars-LimE $\Delta$ coil (red), a marker of F-actin. (F) Typical dynamics of the cortical enrichment of the Factin marker mRFPmars-LimE $\Delta$ coil (top row, red) and GBD probe (bottom row, yellow), in a cell that coexpresses the two probes, upon stimulation with folic acid. (G) Time courses of the cortical enrichment of mRFPmars-LimE $\Delta$ coil $(\boldsymbol{\square})$, and GBD probe $(\diamond)$, in cells that co-express the two probes upon application of $50 \mu \mathrm{M}$ folic acid. The red dashed curve superimposed with the mRFPmars-LimE $\Delta$ coil curve corresponds to the cortical contrast values for GBD probe (lower curve) multiplied by the ratio between the cortical contrast for mRFPmarsLimE $\Delta$ coil and the cortical contrast for GBD probe avaraged over all time points. Shown results are averaged over six cells from at least three independent experiments (mean \pm s.e.m.). Acquisition times of images in $\mathrm{F}$ correspond to every other measurement points in $\mathrm{G}$, starting at the 0 second time point. Scale bars: $5 \mu \mathrm{m}$ (A,C,D); $10 \mu \mathrm{m}$ (B,E,F). Time spans: 325 seconds (A); 120 seconds (B); 210 seconds (C); 200 seconds (D).
This result prompted us to construct a cell line that simultaneously expresses two direct interaction partners of Rac1-GTP: mRFPmars-DGAP1 (referred to as DGAP1 probe hereafter) and GBD probe. Notably, during migration, when these cells adopted a polarized shape, the two fluorescent markers segregated into two non-overlapping cortical compartments. Whereas the GBD probe was clearly enriched at the leading edge, the DGAP1 probe labelled the lateral and posterior cell cortex (Fig. 6B; supplementary material Movie 2). During random motility, Dictyostelium cells occasionally acquire a non-polarized, rounded shape. Cells recorded in such stages showed enrichment of DGAP1 probe throughout the cortex and no cortical enrichment of the GBD probe, thus indicating that the two probes detect two distinct Rac1 populations (Fig. 6C).

\section{GBD probe and DGAP1 probe show opposite localization and dynamics in actin-driven processes}

To further characterize the inter-relationship between the GBD and DGAP1 probes, we monitored cells that express both probes during cytokinesis and phagocytosis. In both processes, the two probes were mutually exclusive. At the onset of cytokinesis, the DGAP1 probe occupied the entire cortex and was gradually restricted to the furrow region, whereas the GBD probe occasionally appeared at the leading edges of incipient daughter cells only at the time of scission (Fig. 7A). In phagocytosis, the DGAP1 probe was excluded from the phagocytotic cups demarcated by GBD probe (Fig. 7B; supplementary material Movie 3). Stimulation with folic acid revealed an opposite dynamics of the cortical enrichment of the two probes. Whereas the GBD probe was recruited to the cortex of the DGAP1-overexpressing cells with a similar dynamics as observed in wild-type cells, DGAP1 probe was, on the contrary, displaced from the cortex with an analogous dynamics (Fig. 7C,D). Because this result indicated that the two probes compete for the same population of activated Rac1, we expressed the GBD probe in cells devoid of DGAP1. Compared with wild-type cells, cortical enrichment of the GBD probe upon chemoattractant stimulation was indeed remarkably stronger in DGAP1-null cells, whereas in DGAP1-overexpressing cells it was weaker than in the wild type (Fig. 7C,D). We also checked localization of GBD probe in DGAP1-null cells during random movement, endocytosis and cytokinesis, and found its spatial distribution not altered compared with distribution in wild-type cells (data not shown).

Finally, we monitored localization of the GBD and DGAP1 probes in chemotaxis. Owing to its stronger cortical enrichment in aggregation-competent DGAP1-null cells, the GBD probe was found to localize to the leading edge of these cells during chemotaxis to cAMP (Fig. 7E; supplementary material Movie 4). As in other actin-driven processes, localization of the DGAP1 probe in chemotaxis was just the reverse: it was enriched at the trailing edge of aggregation-competent cells that express both probes (Fig. 7F; supplementary material Movie 5). Taken together, these results strengthened our conclusion that the GBD and DGAP1 probes detect two distinct populations of activated Rac1 in living Dictyostelium cells. 

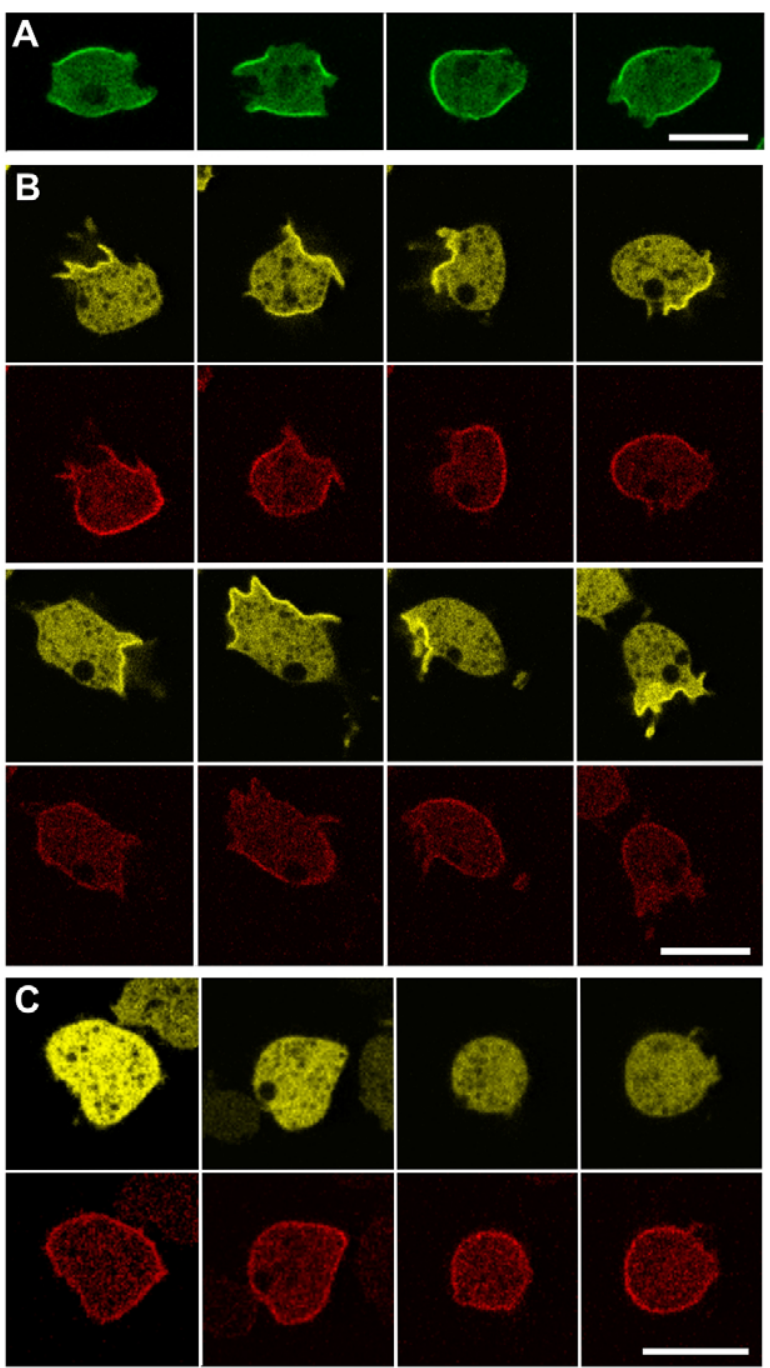

Fig. 6. Localization of fusion proteins in polarized and non-polarized cells. (A) Localization of fusion protein GFP-CI(352-444) in cortexillin-Inull cells during random movement. (B) Opposite localization of the GBD probe (yellow) and the DGAP1 probe (red) in a polarized Dictyostelium cell that expresses both fusion proteins. (C) Four Dictyostelium cells that simultaneously express the GBD probe (yellow) and DGAP1 probe (red) were recorded in non-polarized phases during random movement. Scale bars: $10 \mu \mathrm{m}$. Time spans: 280 seconds (A); 250 seconds (B).

\section{Cortical localization of GBD probe is abolished by treatment with latrunculin $B$}

Prompted by the different behaviour of the two Rac1-GTPbinding probes in both polarized and non-polarized cells, we next examined the intracellular localization of the two probes upon disruption of the actin cytoskeleton with latrunculin B. Shortly after addition of $20 \mu \mathrm{M}$ latrunculin $\mathrm{B}$, the GBD probe was completely removed from the cortical layer of treated cells (Fig. 8A, left column). However, the DGAP1 probe was retained in the cortical layer and in the small spherical blebs that typically form after latrunculin treatment (Fig. 8A, middle column). Localization to these membranous remnants indicates that the DGAP1 probe stays firmly attached to the plasma membrane irrespective of the absence of cortical F-actin. By contrast, a fluorescently labelled probe for F-actin, mRFPmars-ABD120, disappeared from the cortex upon treatment with latrunculin $\mathrm{B}$, as expected, except from a few small remaining nodules (Fig. 8A, right column). All these changes were reversible after removal of latrunculin B. These results indicate that cortical localization of the DGAP1-containing quaternary complex is independent of its cortexillin-mediated binding to F-actin, suggesting that the complex binds to the cell plasma membrane. Cortical localization of the population of activated Rac1 that is recognized by GBD probe, however, critically depends on the integrity of the actin cytoskeleton.

\section{Discussion}

Similarly to motile animal cells such as fibroblasts and leukocytes, Dictyostelium cells harbour a rich repertoire of Rho family GTPases as well as their regulators and downstream effectors (Rivero and Somesh, 2002). Rac is considered to be the founder member of the Rho family (Boureux et al., 2007), which is exemplified in Dictyostelium cells that lack Rho GTPases from the other two groups: Rho and Cdc42. Functional analysis of Dictyostelium Rho GTPases thus offer a useful complement to studies conducted in other cells. Biological roles of some Dictyostelium Rho GTPases have been assessed by genetic knockout and overexpression experiments, as well as localization studies, and a number of their effectors have been identified (Vlahou and Rivero, 2006). RacE, for instance, plays a role in the regulation of cytokinesis (Larochelle et al., 1997), RacB is involved in the control of directional sensing (Park et al., 2004), whereas RacG is important for cell shape, motility and phagocytosis (Somesh et al., 2006). However, direct evidence for the sites of activity and dynamics of RhoGTPases in Dictyostelium has been lacking so far.

Here we investigated the dynamics of activated Rac1 GTPases in Dictyostelium cells using the biosensor approach. Dictyostelium Rac1A, Rac1B and Rac1C are most closely related to human Rac1, and all of them share an identical effector domain (Dumontier et al., 2000). Their general importance for regulation of the actin cytoskeleton was previously demonstrated by stable expression of constitutively active Rac1A and Rac1C, which resulted in dominant-negative effects on actin-based processes including cell migration, endocytosis and cytokinesis, as well as in atypical cell morphology (Dumontier et al., 2000). Similar effects have also been induced by overexpression of constitutively active Rac1B (Duleh et al., 2005). By monitoring the dynamics of two fluorescently labelled Rac1 effectors, PAK1 GBD and DGAP1, in live cells, we now obtained experimental evidence showing that active Rac1 GTPases are involved in the regulation of multiple processes. Most notably, active Rac1 GTPases are simultaneously involved in distinct processes at two opposite sides of migrating cells, protrusion at the front and retraction at the back.

Our results indicate that the posterior activity of Rac1 is mediated by the quaternary cortical complex consisting of the GTPase, IQGAP-related protein DGAP1 and the heterodimeric actin-bundling protein cortexillin. This complex supports cytokinesis and critically determines the mechanical properties and morphology of Dictyostelium cells (Simson et al., 1998; Weber et al., 1999a; Weber et al., 1999b). The most likely candidate for a Rac1 effector at the cell front is the heteropentameric Scar/WAVE complex, which regulates Arp2/3-driven actin polymerization at the leading edge (Insall et al., 2001; Linkner et al., 2011; Pollitt and Insall, 2009; Pollitt et al., 2006). Another possible candidate for a Rac1-GTP-interacting protein at 
A
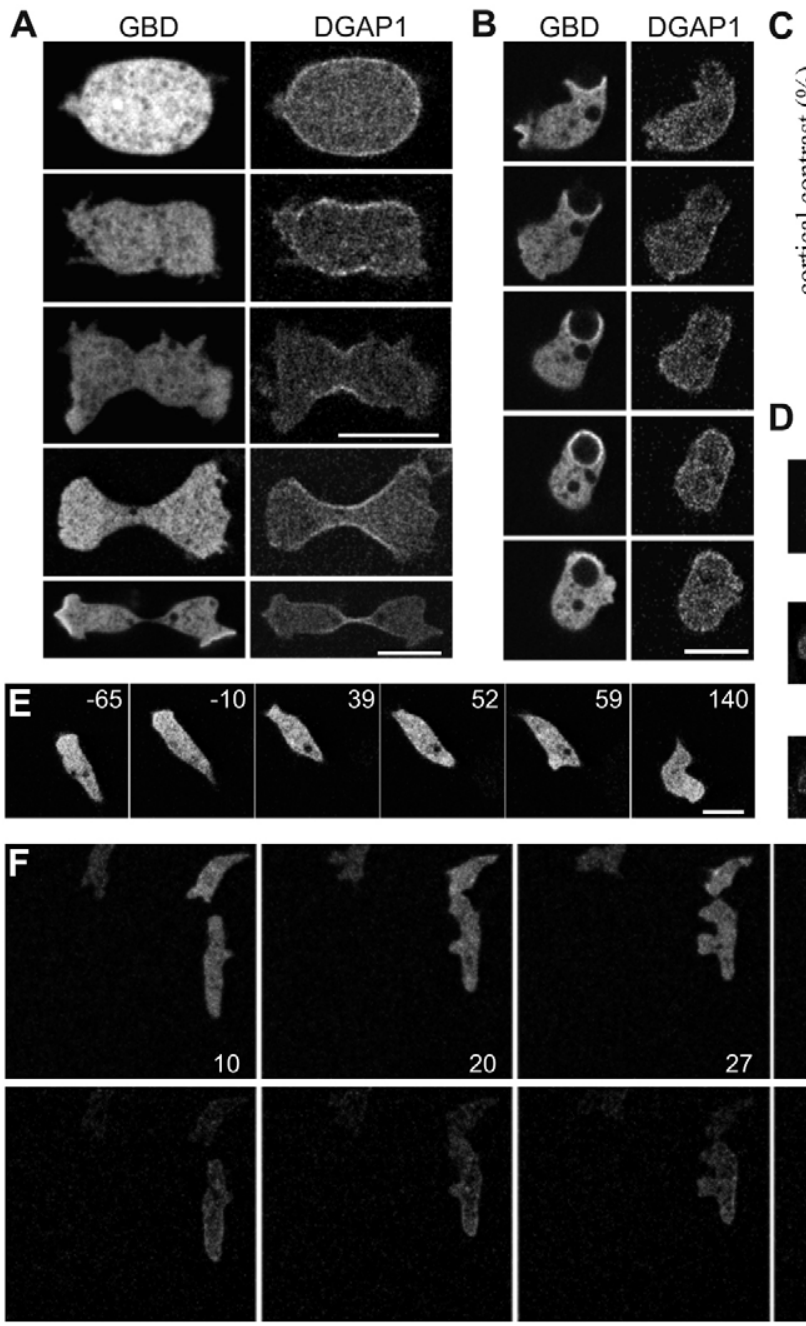
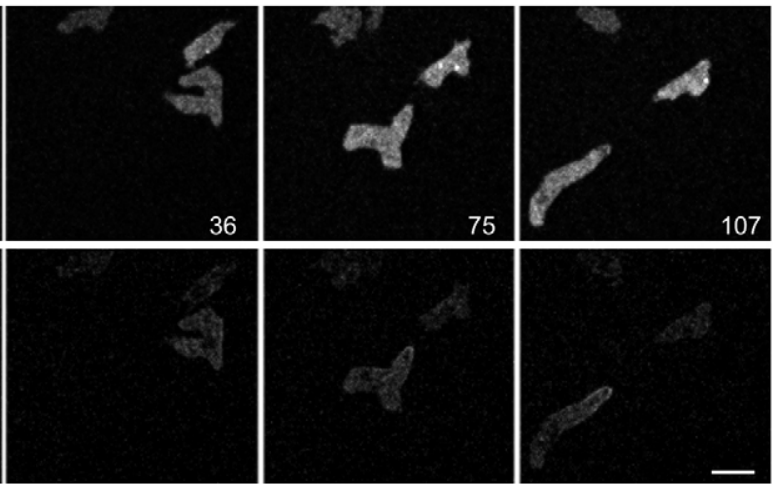

Fig. 7. Localization of the GBD and DGAP1 probes in Dictyostelium. (A) Localization of GBD probe (left column) and DGAP1 probe (right column) in successive phases of cytokinesis in five cells that co-express the two probes. (B) Localization of GBD probe (left column) and DGAP1 probe (right column) during phagocytosis in a cell that co-expresses the two probes. Time span: 50 seconds. (C) Time courses of the cortical enrichment of GBD probe in wild-type cells $(\bullet$, dashed line, reproduced from Fig. 4B as a reference), GBD probe in DGAP1-null cells $(\bullet$, middle curve), GBD probe in DGAP1-overexpressing cells ( $\square$, bottom curve), and DGAP1 probe in DGAP1-overexpressing cells ( $\square$, top curve) upon application of $50 \mu \mathrm{M}$ folic acid. Times in seconds relate to the timepoint of application of the pulse. Results are averaged for six cells from at least three independent experiments (mean \pm s.e.m.). (D) Typical dynamics of the cortical enrichment of GBD probe in DGAP1-null cells (top row), GBD probe in DGAP1-overexpressing cells (middle row), and DGAP1 probe in DGAP1overexpressing cells, upon stimulation with folic acid. Acquisition times of images in D correspond to measurement points in C. (E) Re-distribution of GBD probe in an aggregation competent DGAP1-null cell exposed to a cAMP gradient by means of a micropipette. The micropipette tip was initially positioned $25 \mu \mathrm{m}$ away from the top left corner of the displayed frame, in the 11 o'clock position, and than re-positioned $25 \mu \mathrm{m}$ away from the bottom right corner of the displayed frame, at 5 o'clock. (F) Re-orientation of an aggregation competent cell that co-expresses GBD probe (top row) and DGAP1 probe (bottom row). The micropipette tip was positioned $60 \mu \mathrm{m}$ away from the bottom left corner of the displayed frame, at $8 \mathrm{o}$ 'clock. Times in seconds relate to the time-point of replacement (E), or initial placement (F), of the micropipette. Scale bars: $10 \mu \mathrm{m}$.

the leading edge is formin $\mathrm{C}$ because it has been previously found to accumulate in macropinosomes and phagocytotic cups at the cell front (Kitayama and Uyeda, 2003). Yet another Rac1-GTP effector is DPAKa, but the full-length kinase has not been found to localize to the front of migrating Dictyostelium cells (MüllerTaubenberger et al., 2002).

The GBD probe and DGAP1 probe, which both interact with activated Rac1 in vitro, apparently detect two separate populations of Rac1 GTPases in living cells. At the back of the migrating cells, a proportion of Rac1 is probably bound to a fairly stable complex with DGAP1 and cortexillin. Although formation of this complex depends on interaction between activated Rac1A and DGAP1 (Faix et al., 2001), the exact state of Rac1 inside the assembled complex is not known. It might be in a GTP-bound or a GDPbound form, or might even assume a nucleotide-free form as shown for Cdc42 in a complex with IQGAP1 (Grohmanova et al., 2004). Either way, the complex sequesters Rac1 at the back of polarized cells and probably leaves a small number of activated Rac1 molecules accessible to GBD probe. A much faster Rac1 turnover at the front renders its GTP-bound form more accessible to a transient binding of GBD probe (Fig. 8B). Despite a mutually exclusive localization of the two probes, the extent of the free 
A

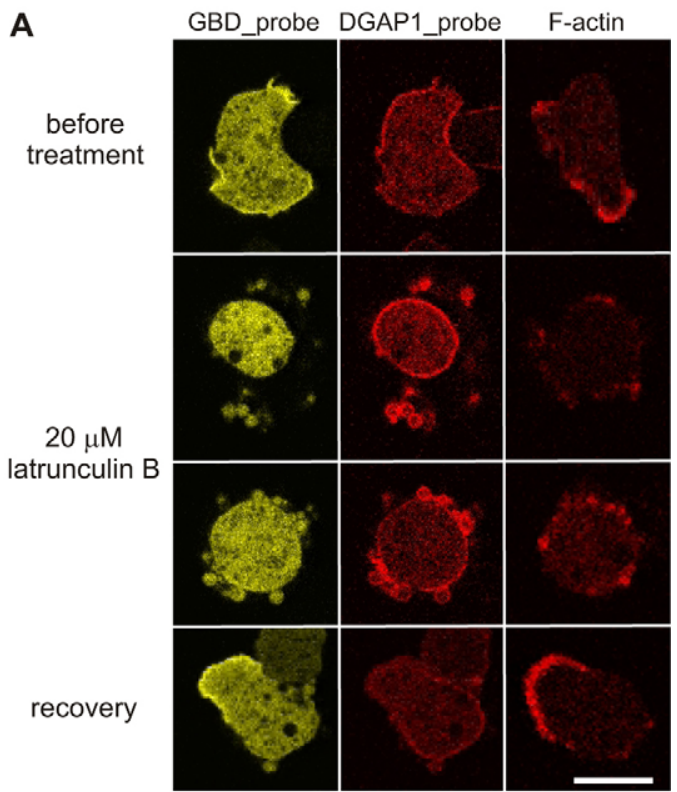

B

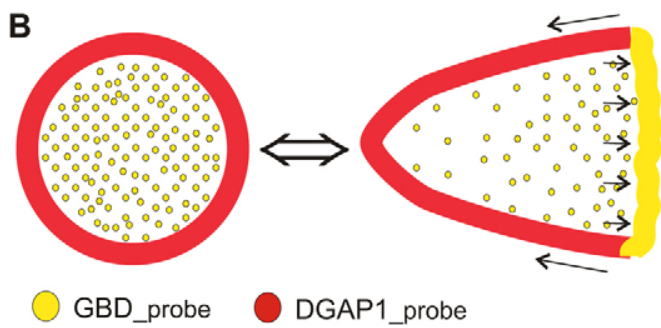

Fig. 8. Effect of latrunculin B on cortical localization of the GBD and DGAP1 probes. (A) Cells that co-express the GBD (yellow, left column) and DGAP1 probes (red, middle column) before treatment with latrunculin B (top row), 20 minutes after addition of $20 \mu \mathrm{M}$ latrunculin $\mathrm{B}$ (two middle rows), and 30 minutes after the washout (bottom row). The right column shows cells that express an F-actin marker, the actin-binding domain ABD from ABP 120 $\mathrm{kDa}$ protein fused with mRFPmars. Scale bar: $10 \mu \mathrm{m}$. (B) Schematic presentation of the intracellular distribution of two Rac1 effectors, GBD probe (yellow) and DGAP1 probe (red), in non-polarized (left) and polarized (right) cells. In non-polarized cells, DGAP1 probe is localized to the entire cell cortex, whereas GBD probe remains in the cytoplasm. In polarized cells, GBD probe is recruited to the protruding leading edge, and DGAP1 probe is displaced from that cortical region.

Rac1-GTP zone appears not to be limited by the DGAP1 complex, because it is unchanged in DGAP1-null mutants. However, another IQGAP-related protein from Dictyostelium, GAPA, has partly overlapping functions with DGAP1 (Faix et al., 2001), and might play a role in restricting the Rac1-GTP zone labeled by the GBD probe in the absence of DGAP1.

The Rac1-sequestering function of the DGAP1-cortexillin complex is supported by its chemoattractant-induced disassembly and release from the cortex, coupled with the cortical recruitment of free Rac1A (Fig. 7). In polarized cells, the Rac1-sequestering activity of the complex appears to suppress membrane protrusions driven by actin polymerization in the rear of cells. The importance of the DGAP1-containing complex for regulation of cell migration and phagocytosis is manifested in phenotypes of DGAP1-null and DGAP1-overexpressing mutants (Faix et al., 1998). DGAP1overexpressing cells are making fewer actin-based protrusions, move more slowly and phagocytose less efficiently than wild-type cells, which is consistent with more Rac1 being trapped inside the DGAP1 complex and therefore not available to promote actinbased motility. DGAP1-null cells, on the contrary, are making more actin-based protrusions and move faster than wild-type cells Consistently, it has also been shown that lack of DGAP1 leads to an increased cellular F-actin content (Faix et al., 1998).

Our finding of distinct and non-overlapping localizations of two Rac effector proteins is not without precedence. Distinct patterns of colocalization of Rac1 with its two effectors, IQGAP1 and Sra-1, at the inner side of the ventral membrane of Vero cells was determined by double immunogold labelling (Watanabe et al., 2008). Closely related to DGAP1, IQGAP1 is also involved in crosslinking of actin filaments, whereas Sra-1 is a component of the hetero-pentameric Scar/WAVE regulatory complex, which induces formation of lamellipodia (Chen et al., 2010). Similarly, distinct spatial association patterns between $\mathrm{Cdc} 42$ and its key effector proteins, PAK1 and N-WASP, were revealed by multiphoton fluorescence lifetime imaging microscopy in breast carcinoma cells (Parsons et al., 2005).

Evidence for additional roles of Rac beyond its involvement in protrusion of the leading edge was obtained using a FRET-based biosensor for Rac activity in living cells, where activated Rac was detected in the retracting tails of migrating neutrophils (Gardiner et al., 2002). Consistently, Rac1-deficient neutrophils were reported to display an inability to retract their tails (Sun et al., 2004). Ectopic expression of members of the Rac subfamily not only triggered the formation of lamellipodia but also induced the formation of bundles of actin filaments (Aspenström et al., 2004). Finally, Rac1 was shown not only to inhibit Rho signalling at the leading edge but also to positively regulate the function of RhoA-myosin-II at the back of chemotactic neutrophils (Pestonjamasp et al., 2006). In the present work, we provide yet another example of Rac activity at both ends of polarized cells, supporting multiple signalling roles of Rac GTPases in the control of cell migration.

Localization of DGAP1 probe at the cell boundary is independent of F-actin, which indicates that the quaternary posterior complex is probably bound to the plasma membrane by the phosphatidylinositol-4,5-bisphosphate-binding C-terminal domain of cortexillin I. This C-terminal nonapeptide has been shown to almost double the accumulation of cortexillin I in the cell cortical layer and to suppress the actin-bundling activity of cortexillin I when bound to phosphatidylinositol-4,5bisphosphate (Stock et al., 1999). Posterior localization of the quaternary complex might be mediated by phosphatase and tensin homolog (PTEN), which converts phosphatidylinositol3,4,5-trisphosphate to phosphatidylinositol-4,5-bisphosphate, because PTEN is also enriched at the lateral and rear regions of migrating cells (Arai et al., 2010). However, sensitivity of the cortical localization of GBD probe to latrunculin B treatment suggests that the anterior Rac1-GTP is not stably anchored to the membrane in the absence of an actin cortex, or that the cortical localization of a RacGEF responsible for Rac1 activation depends on F-actin. Interestingly, cortical localization of a member of the Dictyostelium Dock180 family of RacGEFs, DockD, indeed depends on actin polymerization (Para et al., 2009). A related example is provided by the WAVE complex in neutrophils, whose proper assembly and localization also rely on integrity of the actin cytoskeleton (Millius et al., 2009). Unlike the DGAP1 probe, the GBD probe is not enriched in the cortex of round, non-polarized cells that have an intact cortical actin layer 
(Fig. 6C). It appears therefore that the default, symmetrical state of the cortex in Dictyostelium cells is determined by the activity of the posterior quaternary complex, whereas breaking of symmetry is brought about by an anterior activity, which leads to cell polarization.

By the use of Rac1A(WT FRET $\left._{\text {F }}\right)$ probe, RacGEF activity was detected throughout the cell cortex and in the perinuclear region of transfected cells (Fig. 3). FRET sensors used in this work are designed to indicate the presence of RacGEF proteins that facilitate the exchange of GDP by GTP on Rac1A, and thus drive interaction between activated GTPase and its effector PAK1 GBD within the probe. However, these sensors cannot discriminate between different RacGEFs and will, therefore, report Rac1A activation irrespective of a particular GEF activity and its specific interactions. Only by using two probes, a GBD probe and a DGAP1 probe, which are designed to interact with endogenous Rac1-GTP but based on different Rac1 effectors, did it become possible to pin down two non-overlapping dynamic populations of Racl that are probably activated by different GEFs. RacGEFs responsible for activation of Rac1 at the cell front probably belong to the Dictyostelium Dock180 family, because DockD was shown to form a complex with DdELMO1 and Rac1A (Para et al., 2009). A counterpart of Dock180 GEFs at the cell back remains to be identified.

Our findings lead us to propose a novel mechanism for spatial and temporal regulation of Rac activity by competing effector proteins. Our results imply that two spatially separated effector complexes allow for retention and selective recruitment of Rac to functionally distinct cortical regions. Thereby, a high abundance of an activated GTPase, as exemplified by Rac1 in Dictyostelium, can be maintained in the cortex and facilitates a fast and localized response to upstream signals (Postma et al., 2004). In conclusion, Rac1 GTPases appear to be key mediators in signalling cascades that lead to both protrusion at the front and retraction at the back of Dictyostelium cells. The tools described in this report will be instrumental to further explore spatial and temporal relationships between different signalling pathways and morphological changes in these small and fast-moving cells.

\section{Materials and Methods}

\section{Cell culture, cell lines and transformation of $D$. discoideum cells}

All $D$. discoideum cell lines used in this study were cultivated in axenic medium at $23^{\circ} \mathrm{C}$ on polystyrene culture dishes as previously described (Faix et al., 2001). Cell transformation by electroporation and clonal selection was performed as described (Kimmel and Faix, 2006). Cell lines were derived from AX2 parent strain and express the following constructs: GFP-Rac1A(WT), GFP-Rac1A(V12), GFPRac1A(N17) (Dumontier et al., 2000), GFP-RacC(WT) (this work), PAK1(GBD)DYFP (this work), PAK1(GBD)-DYFP and mRFPmars-DGAP1 (this work), PAK1(GBD)-DYFP and mRFPmars-Rac1A(WT) (this work), PAK1(GBD)DYFP and mRFPmars-LimE $\Delta$ coil (this work, derived from AX2/mRFPmarsLimEAcoil) (Schneider et al., 2003), DPAKa(GBD)-DYFP (this work) and mRFPmars-ABD120 (Fischer et al., 2004). In this study, we used DYFP (MüllerTaubenberger, 2006) and mRFPmars (Fischer et al., 2004) fluorescent proteins that were adapted for the codon usage of $D$. discoideum cells. All unimolecular probes for FRET were also expressed in the AX2 parent strain (this work, see Table 1). Cortexillin-I-null mutants expressing GFP-CI(aa 352-444), $\mathrm{CI}^{-} / \mathrm{GFP}-\mathrm{CI}(352-$ 444), were described previously (Weber et al., 1999a). PAK1(GBD)-DYFP was also expressed in a DGAP1-null strain (Faix et al., 1998).

\section{D. discoideum transformation vectors}

cDNAs encoding PAK1(GBD)-DYFP and GFP-RacC(WT) were cloned from auxiliary vectors into the HindIII site of pDEXH and into the EcoRI and HindIII sites of pDEXRH expression vectors, respectively (Faix et al., 1992). The orientation of the PAK1(GBD)-DYFP insert was confirmed by digestion with $B g l$ II and Asp718. For expression of a DPAKa(GBD)-DYFP construct, cDNA encoding the DPAKa GBD was amplified from auxiliary vector by PCR and cloned into the BamHI and SalI sites of pDEXRH-MCS-DYFP, a vector derived from pDEXRH. The pDEXRH-MCS-DYFP was constructed by introduction of multiple cloning sites and DYFP into the EcoRI and HindIII sites of pDEXRH. Expression vectors for mRFPmars-DGAP1 and mRFPmars-Rac1A(WT) fusion proteins were generated by insertion of cDNA encoding DGAP1 and Rac1A(WT) into the BamHI and EcoRI sites, and BamHI and ClaI sites, of plasmid p339-3, respectively (Fischer et al., 2004). All vectors encoding unimolecular FRET probes are based on pDEXRH, and the cloning procedure closely followed construction of the Raichu-Rac probe described previously (Itoh et al., 2002). For

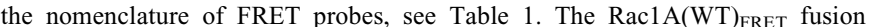
protein consists of DYFP (aa 1-238), a spacer (Ile-Asp), GBD from rat PAK1 (aa 57-200), a spacer (Ser-Gly-Gly-Thr-Gly-Gly-Gly-Gly-Ser), D. discoideum Rac1A (aa 1-176), a spacer (Gly-Gly-Arg-Val-Asp), mRFPmars (aa 1-225), a spacer (Gly-Arg-Ser-Arg) and D. discoideum Rac1A (aa 177-194) (Fig. 3A). In

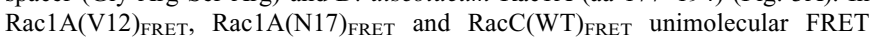
probes, Rac1A (aa 1-176) was replaced with active Rac1A(V12) (aa 1-176), inactive Rac1A(N17) (aa 1-176), or the wild-type form of RacC (aa 1-179), respectively. Triple fusion protein for detection of endogenous activated Rac1

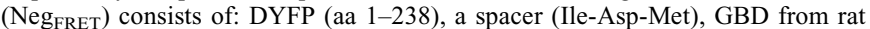
PAK1 (aa 57-200), a spacer (Ser-Gly-Gly-Thr-Gly-Gly-Gly-Gly-Ser-Val-Asp), mRFPmars (aa 1-225), a spacer (Gly-Arg-Ser-Arg) and Rac1A (aa 177-194). Unimolecular probe POSFRE was used as a positive control for intracellular FRET between DYFP and mRFPmars: DYFP (aa 1-238), a spacer (Gly-Ser-GlyGly-Thr-Gly-Gly-Gly-Gly-Thr), mRFPmars (aa 1-225) and Rac1A (aa 177-194).

\section{Yeast two-hybrid assay}

For yeast two-hybrid (Y2H) assay, we used Matchmaker GAL4 Two-Hybrid System 3 (Clontech Laboratories). cDNAs encoding DGAP1 (aa 1-822), DGAP1 (aa 161-822), DPAKa (aa 731-890) and PAK1 (aa 57-200) were inserted into the $E c o$ RI and BamHI sites of pGADT7 vector. cDNAs encoding constitutively active forms of 11 Dictyostelium Rac GTPases [Rac1A(G12V), Rac1C(G12V), $\operatorname{RacB}(\mathrm{G} 12 \mathrm{~V}), \quad \operatorname{RacC}(\mathrm{G} 15 \mathrm{~V}), \quad \operatorname{RacD}(\mathrm{G} 17 \mathrm{~V}), \quad \operatorname{RacE}(\mathrm{G} 20 \mathrm{~V}), \quad \operatorname{RacF} 1(\mathrm{G} 12 \mathrm{~V})$, RacG(G12V), RacH(M13V), RacI(S14V) and RacJ(D18V)] and constitutively inactive forms of three GTPases [Rac1A(T17N), Rac1C(T17N) and RacC(T20N)] were cloned into the EcoRI and SalI sites of pGBKT7 vector. Constructs were introduced into yeast strain AH109 and interactions of expressed proteins estimated according to the growth on selective media at low and high stringency after 3 days at $30^{\circ} \mathrm{C}$, as indicated in Fig. 2A. Control interactions were verified according to instructions of the manufacturer.

Protein expression and production of polyclonal anti-PAK1 GBD antibodies The cDNA of the PAK1 GBD domain (aa 57-200) was fused, in one or two copies, to GST cDNA using pGEX-6P-1 plasmid (GE Healthcare), resulting in two expression plasmids that encode GST-PAK1(GBD) or GST-[PAK1(GBD) $]_{2}$. A plasmid encoding GST-Rac1A(WT) fusion protein has been described previously (Faix et al., 1998). All GST fusion proteins were expressed and purified from E. coli strain Rosetta by standard procedures and subsequently dialysed against PBS containing $140 \mathrm{mM} \mathrm{NaCl}, 2.7 \mathrm{mM} \mathrm{KCl}, 1 \mathrm{mM} \mathrm{MgCl} 2,10 \mathrm{mM} \mathrm{Na}_{2} \mathrm{HPO}_{4}$ and $1.8 \mathrm{mM} \mathrm{KH}_{2} \mathrm{PO}_{4}$, pH 7.3 (Faix et al., 1998). The GBD of PAK1 was expressed as a fusion with maltosebinding protein (MBP) using plasmid pMAL-c2X (New England Biolabs). MBPPAK1(GBD) was expressed and purified from E. coli strain Rosetta by amylose agarose affinity chromatography according to the manufacturer's instructions. GSTPAK1(GBD) was used as an antigen for production of polyclonal anti-PAK1 GBD antibodies using female white New Zealand rabbits (Sigma). Antibodies against PAK1 GBD were affinity purified from serum using MBP-PAK1(GBD) covalently bound to cyanogen bromide-activated-Sepharose 4B (Sigma). The specificity of the purified antibodies was assessed by western blotting of total cellular proteins of AX2 cells expressing DYFP-tagged PAK1 GBD.

\section{GST-fusion protein binding assays}

All GST-binding assays were performed essentially as described previously (Faix et al., 1998). Briefly, GST-[PAK1(GBD) $]_{2}$ and GST-Rac1A(WT) fusion proteins were bound to glutathione-Sepharose beads. Beads with GST-[PAK1(GBD) $]_{2}$ were separately incubated with lysates of AX2 cells expressing GFP-Rac1A(WT), GFP-Rac1A(V12), GFP-Rac1A(N17) or GFP-RacC(WT). After elution of bound proteins with hot SDS sample buffer, interactions between PAK1 GBD and the assayed Rac proteins were determined by western blotting using polyclonal antiGFP antibodies (Faix et al., 2001). GST-Rac1A(WT) bound to Sepharose was loaded with either GDP or non-hydrolysible GTP analogue GTP $\gamma \mathrm{S}$ and then used to pull down PAK1(GBD)-DYFP from lysate of AX2/PAK1(GBD)-DYFP cells. The bound proteins were eluted with SDS sample buffer and probed in Western blots by polyclonal antibodies against PAK1 GBD.

\section{Fluorescence microscopy and functional assays}

Fluorescence microscopy of living Dictyostelium cells was carried out on a confocal microscope Leica TCS SP2 AOBS (Leica Microsystems) at $23^{\circ} \mathrm{C}$, in 
small chambers assembled on glass coverslips (Weber et al., 1995). Cells were washed twice in Sörensen phosphate buffer 30 minutes before scanning. For phagocytosis assays, cells were mixed with TRITC-labelled yeast particles, and for monitoring cytokinesis, Klebsiella aerogenes bacteria were added as described (Weber et al., 1999a). For disruption of the actin cytoskeleton, cells were exposed for 20 minutes to $20 \mu \mathrm{M}$ latrunculin B (Sigma). For chemotaxis assays, cells were washed twice and incubated in phosphate buffer on Petri dishes for at least 6 hours at a near-confluent density, until cell stream formation commenced. Chemotaxis of single cells was induced by a micropipette (Femtotip II, Eppendorf) filled with $100 \mu$ M cAMP (Sigma) (Weber et al., 1995). Uniform stimulation of vegetative cells with folic acid was performed by rapidly adding $10 \mu \mathrm{l}$ of $250 \mu \mathrm{M}$ folic acid solution to $40 \mu \mathrm{l}$ of cell suspension in phosphate buffer. Uniform stimulation with cAMP was performed by rapidly adding $10 \mu 1$ of $150 \mu \mathrm{M}$ cAMP solution to $40 \mu$ of cell suspension in the phosphate buffer. For cAMP stimulation experiments, we used early-stage aggregation competent cells after 5 hours of starvation. Cortical contrast was defined as the following ratio of average fluorescence intensities (I) in the cortex and the cytoplasm: $[I($ cortex $)-I($ cytoplasm $)] / I($ cytoplasm $)$. The fluorescence intensities were determined using the QuimP10 plug-in module for ImageJ (Dormann et al., 2002). In the module, thickness of the cortex was set to $2 \mu \mathrm{m}$, and cortical intensity determined at 200 node points per cell. The excitation wavelengths and detection ranges used for imaging were $488 \mathrm{~nm}$ and $500-560 \mathrm{~nm}$ for GFP, $514 \mathrm{~nm}$ and $525-565 \mathrm{~nm}$ for DYFP, and $594 \mathrm{~nm}$ and $605-670 \mathrm{~nm}$ for mRFPmars

\section{Fluorescence resonance energy transfer (FRET)}

Sensitized emission of the acceptor (mRFPmars) was recorded upon excitation of the donor (DYFP) in live $D$. discoideum cells, using Leica Application Wizard for FRET Sensitized Emission (Leica Microsystems). Signals in three channels (donor, acceptor and FRET) were acquired by sequential scanning with 514 and $594 \mathrm{~nm}$ laser lines. Channel settings (excitation/detection) were as follows: donor $(514 \mathrm{~nm} / 525-560 \mathrm{~nm})$; acceptor $(594 \mathrm{~nm} / 605-670 \mathrm{~nm})$; FRET $(514 \mathrm{~nm} / 605$ $670 \mathrm{~nm})$. For correction of bleed-through and cross-excitation effects, cells that express FRET probes were mixed with cells that express a pure donor species (AX2/DPAKa(GBD)-DYFP), and cells that express a pure acceptor species (AX2/ mRFPmars-ABD120). At least one cell of each species was present in each recorded image (Fig. 3C). Image processing and quantification of FRET were performed using the PixFRET plug-in module for ImageJ (Feige et al., 2005) Apparent FRET efficiency was evaluated only for pixels belonging to the cell cortex using the Histogram Selection Tool of the PixFRET plug-in. Average cortical FRET efficiency was calculated for at least ten cells of each investigated strain and further averaged for each strain (mean \pm s.d.). Pair-wise statistical comparison between strains was performed using the two-tailed $t$-test.

\section{Acknowledgements}

We thank Annette Müller-Taubenberger for AX2/mRFPmarsLimE $\Delta$ coil and AX2/mRFPmars-ABD120 cells, and Alfred Wittinghofer for rat $P A K 1 \mathrm{cDNA}$.

\section{Funding}

This work was supported by the Unity through Knowledge Fund [grant number 1A 10/07 to I.W.]; the Ministry of Science, Education and Sport of the Republic of Croatia [grant number 098-0982913-2858 to I.W.]; Deutsche Akademische Austauschdienst [grant number DAADD/07/00065 to J.F.]; and Deutsche Forschungsgemeinschaft [grant number DFG FA 330/4-2 to J.F.].

\section{Supplementary material available online at}

http://jcs.biologists.org/lookup/suppl/doi:10.1242/jcs.089680/-/DC1

\section{Reference}

Arai, Y., Shibata, T., Matsuoka, S., Sato, M., Yanagida, T. and Ueda, M. (2010) Self-organization of the phosphatidylinositol lipids signaling system for random cell migration. Proc. Natl. Acad. Sci. USA 107, 12399-12404.

Aspenström, P., Fransson, A. and Saras, J. (2004). Rho GTPases have diverse effects on the organization of the actin filament system. Biochem. J. 377, 327-337.

Boureux, A., Vignal, E., Faure, S. and Fort, P. (2007). Evolution of the Rho family of Ras-like GTPases in eukaryotes. Mol. Biol. Evol. 24, 203-216.

Buchwald, G., Hostinova, E., Rudolph, M. G., Kraemer, A., Sickmann, A., Meyer, H. E., Scheffzek, K. and Wittinghofer, A. (2001). Conformational switch and role of phosphorylation in PAK activation. Mol. Cell. Biol. 21, 5179-5189.

Chen, Z. C., Borek, D., Padrick, S. B., Gomez, T. S., Metlagel, Z., Ismail, A. M., Umetani, J., Billadeau, D. D., Otwinowski, Z. and Rosen, M. K. (2010). Structure and control of the actin regulatory WAVE complex. Nature 468, 533-538.

DerMardirossian, C. and Bokoch, G. M. (2005). GDIs: central regulatory molecules in Rho GTPase activation. Trends Cell Biol. 15, 356-363.
Dormann, D., Libotte, T., Weijer, C. J. and Bretschneider, T. (2002). Simultaneous quantification of cell motility and protein-membrane-association using active contours. Cell Motil. Cytoskeleton 52, 221-230.

Duleh, S. N., Collins, J. T. B. and Pope, R. K. (2005). Morphological and functional analysis of Rac1B in Dictyostelium discoideum. J. Electron Microsc. (Tokyo) 54, 519-528.

Dumontier, M., Höcht, P., Mintert, U. and Faix, J. (2000). Rac1 GTPases control filopodia formation, cell motility, endocytosis, cytokinesis and development in Dictyostelium. J. Cell Sci. 113, 2253-2265.

Faix, J. and Grosse, R. (2006). Staying in shape with formins. Dev. Cell 10, 693-706.

Faix, J., Gerisch, G. and Noegel, A. A. (1992). Overexpression of the csA cell adhesion molecule under its own cAMP-regulated promoter impairs morphogenesis in Dictyostelium. J. Cell Sci. 102, 203-214.

Faix, J., Clougherty, C., Konzok, A., Mintert, U., Murphy, J., Albrecht, R., Mühlbauer, B. and Kuhlmann, J. (1998). The IQGAP-related protein DGAP1 interacts with Rac and is involved in the modulation of the F-actin cytoskeleton and control of cell motility. J. Cell Sci. 111, 3059-3071.

Faix, J., Weber, I., Mintert, U., Köhler, J., Lottspeich, F. and Marriott, G. (2001) Recruitment of cortexillin into the cleavage furrow is controlled by Rac1 and IQGAPrelated proteins. EMBO J. 20, 3705-3715.

Feige, J. N., Sage, D., Wahli, W., Desvergne, B. and Gelman, L. (2005). PixFRET, an ImageJ plug-in for FRET calculation that can accommodate variations in spectral bleed-throughs. Microsc. Res. Tech. 68, 51-58.

Fischer, M., Haase, I., Simmeth, E., Gerisch, G. and Müller-Taubenberger, A. (2004). A brilliant monomeric red fluorescent protein to visualize cytoskeleton dynamics in Dictyostelium. FEBS Lett. 577, 227-232.

Gardiner, E. M., Pestonjamasp, K. N., Bohl, B. P., Chamberlain, C., Hahn, K. M. and Bokoch, G. M. (2002). Spatial and temporal analysis of Rac activation during live neutrophil chemotaxis. Curr. Biol. 12, 2029-2034.

Gerisch, G., Bretschneider, T., Müller-Taubenberger, A., Simmeth, E., Ecke, M., Diez, S. and Anderson, K. (2004). Mobile actin clusters and traveling waves in cells recovering from actin depolymerization. Biophys. J. 87, 3493-3503.

Grohmanova, K., Schlaepfer, D., Hess, D., Gutierrez, P., Beck, M. and Kroschewski, R. (2004). Phosphorylation of IQGAP1 modulates its binding to Cdc42, revealing a new type of Rho-GTPase regulator. J. Biol. Chem. 279, 48495 48504 .

Heasman, S. J. and Ridley, A. J. (2008). Mammalian Rho GTPases: new insights into their functions from in vivo studies. Nat. Rev. Mol. Cell Biol. 9, 690-701.

Heasman, S. J., Carlin, L. M., Cox, S., Ng, T. and Ridley, A. J. (2010). Coordinated RhoA signaling at the leading edge and uropod is required for $\mathrm{T}$ cell transendothelial migration. J. Cell Biol. 190, 553-563.

Iden, S. and Collard, J. G. (2008). Crosstalk between small GTPases and polarity proteins in cell polarization. Nat. Rev. Mol. Cell Biol. 9, 846-859.

Insall, R., Müller-Taubenberger, A., Machesky, L., Köhler, J., Simmeth, E., Atkinson, S., Weber, I. and Gerisch, G. (2001). Dynamics of the Dictyostelium Arp2/3 complex in endocytosis, cytokinesis, and chemotaxis. Cell Motil. Cytoskeleton 50, $115-128$

Itoh, R. E., Kurokawa, K., Ohba, Y., Yoshizaki, H., Mochizuki, N. and Matsuda, M. (2002). Activation of Rac and $\mathrm{Cdc} 42$ video imaged by fluorescent resonance energy transfer-based single-molecule probes in the membrane of living cells. Mol. Cell. Biol. 22, 6582-6591.

Kimmel, A. R. and Faix, J. (2006). Generation of multiple knockout mutants using the Cre-loxP system. Methods Mol. Biol. 346, 187-199.

Kitayama, C. and Uyeda, T. Q. P. (2003). ForC, a novel type of formin family protein lacking an FH1 domain, is involved in multicellular development in Dictyostelium discoideum. J. Cell Sci. 116, 711-723.

Larochelle, D. A., Vithalani, K. K. and De Lozanne, A. (1997). Role of Dictyostelium RacE in cytokinesis: mutational analysis and localization studies by use of green fluorescent protein. Mol. Biol. Cell 8, 935-944.

Linkner, J., Witte, G., Stradal, T., Curth, U. and Faix, J. (2011). High-resolution Xray structure of the trimeric Scar/WAVE-Complex precursor Brk1. PLOS ONE 6 e21327 doi: 10.1371/journal.pone.0021327.

Machacek, M., Hodgson, L., Welch, C., Elliott, H., Pertz, O., Nalbant, P., Abell, A., Johnson, G. L., Hahn, K. M. and Danuser, G. (2009). Coordination of Rho GTPase activities during cell protrusion. Nature 461, 99-103.

Millius, A., Dandekar, S. N., Houk, A. R. and Weiner, O. D. (2009). Neutrophils establish rapid and robust WAVE complex polarity in an actin-dependent fashion. Curr. Biol. 19, 253-259.

Müller-Taubenberger, A. (2006). Application of fluorescent protein tags as reporters in live-cell imaging studies. Methods Mol. Biol. 346, 229-246.

Müller-Taubenberger, A., Bretschneider, T., Faix, J., Konzok, A., Simmeth, E. and Weber, I. (2002). Differential localization of the Dictyostelium kinase DPAKa during cytokinesis and cell migration. J. Muscle Res. Cell Motil. 23, 751-763.

Nobes, C. D. and Hall, A. (1995). Rho, Rac, and Cdc42 GTPases regulate the assembly of multimolecular focal complexes associated with actin stress fibers, lamellipodia, and filopodia. Cell $\mathbf{8 1}, 53-62$.

Noegel, A. A. and Schleicher, M. (2000). The actin cytoskeleton of Dictyostelium: story told by mutants. J. Cell Sci. 113, 759-766.

Para, A., Krischke, M., Merlot, S., Shen, Z., Oberholzer, M., Lee, S., Briggs, S. and Firtel, R. A. (2009). Dictyostelium Dock180-related RacGEFs regulate the actin cytoskeleton during cell motility. Mol. Biol. Cell 20, 699-707. 
Park, K. C., Rivero, F., Meili, R., Lee, S., Apone, F. and Firtel, R. (2004). Rac regulation of chemotaxis and morphogenesis in Dictyostelium. EMBO J. 23, 41774189

Parsons, M., Monypenny, J., Ameer-beg, S. M., Millard, T. H., Machesky, L. M., Peter, M., Keppler, M. D., Schiavo, G., Watson, R. and Chernoff, J. et al. (2005). Spatially distinct binding of Cdc42 to PAK1 and N-WASP in breast carcinoma cells. Mol. Cell. Biol. 25, 1680-1695.

Pertz, O. and Hahn, K. M. (2004). Designing biosensors for Rho family proteins deciphering the dynamics of Rho family GTPase activation in living cells. J. Cell Sci. 117, 1313-1318.

Pestonjamasp, K. N., Forster, C., Sun, C., Gardiner, E. M., Bohl, B., Weiner, O., Bokoch, G. M. and Glogauer, M. (2006). Rac1 links leading edge and uropod events through Rho and myosin activation during chemotaxis. Blood 108, 2814-2820.

Pollitt, A. Y. and Insall, R. H. (2009). WASP and SCAR/WAVE proteins: the drivers of actin assembly. J. Cell Sci. 122, 2575-2578.

Pollitt, A. Y., Blagg, S. L., Ibarra, N. and Insall, R. H. (2006). Cell motility and SCAR localisation in axenically growing Dictyostelium cells. Eur. J. Cell Biol. 85, 10911098

Postma, M., Bosgraaf, L., Loovers, H. M. and Van Haastert, P. J. M. (2004). Chemotaxis: signalling modules join hands at front and tail. EMBO Reports 5, 35-40.

Rivero, F. and Somesh, B. P. (2002). Signal transduction pathways regulated by Rho GTPases in Dictyostelium. J. Muscle Res. Cell Motil. 23, 737-749.

Rossman, K. L., Der, C. J. and Sondek, J. (2005). GEF means go: turning on RHO GTPases with guanine nucleotide-exchange factors. Nat. Rev. Mol. Cell Biol. 6, 167180.

Sasaki, A. T., Janetopoulos, C., Lee, S., Charest, P. G., Takeda, K., Sundheimer, L. W., Meili, R., Devreotes, P. N. and Firtel, R. A. (2007). G protein-independent Ras/PI3K/ F-actin circuit regulates basic cell motility. J. Cell Biol. 178, 185-191.

Schneider, N., Weber, I., Faix, J., Prassler, J., Müller-Taubenberger, A., Köhler, J., Burghardt, E., Gerich, G. and Marriott, G. (2003). A Lim protein involved in the progression of cytokinesis and regulation of the mitotic spindle. Cell Motil. Cytoskeleton 56, 130-139.
Simson, R., Wallraff, E., Faix, J., Niewöhner, J., Gerisch, G. and Sackmann, E. (1998). Membrane bending modulus and adhesion energy of wild-type and mutant cells of Dictyostelium lacking talin or cortexillins. Biophys. J. 74, 514-522.

Somesh, B. P., Vlahou, G., Iijima, M., Insall, R. H., Devreotes, P. and Rivero, F. (2006). RacG regulates morphology, phagocytosis, and chemotaxis. Eukaryot. Cell 5, 1648-1663.

Stock, A., Steinmetz, M. O., Janmey, P. A., Aebi, U., Gerisch, G., Kammerer, R. A., Weber, I. and Faix, J. (1999). Domain analysis of cortexillin I: actin-bundling, PIP2binding and the rescue of cytokinesis. EMBO J. 18, 5274-5284.

Sun, C. X., Downey, G. P., Zhu, F., Koh, A. L. Y., Thang, H. and Glogauer, M. (2004). Rac1 is the small GTPase responsible for regulating the neutrophil chemotaxis compass. Blood 104, 3758-3765.

Tcherkezian, J. and Lamarche-vane, N. (2007). Current knowledge of the large RhoGAP family of proteins. Biol. Cell 99, 67-86.

Van Haastert, P. J. M. and Devreotes, P. N. (2004). Chemotaxis: signalling the way forward. Nat. Rev. Mol. Cell Biol. 5, 626-634.

Vlahou, G. and Rivero, F. (2006). Rho GTPase signaling in Dictyostelium discoideum: Insights from the genome. Eur. J. Cell Biol. 85, 947-959.

Wang, L. and Zheng, Y. (2007). Cell type-specific functions of Rho GTPases revealed by gene targeting in mice. Trends Cell Biol. 17, 58-64.

Watanabe, T., Wang, S., Kakeno, M., Usukura, J. and Kaibuchi, K. (2008). Ultrastructural study of Racl and its effectors beneath the substratum-facing membrane. Cell Struct. Funct. 33, 101-107.

Weber, I., Wallraff, E., Albrecht, R. and Gerisch, G. (1995). Motility and substratum adhesion of Dictyostelium wild-type and cytoskeletal mutant cells: a study by RICM/ bright-field double-view image analysis. J. Cell Sci. 108, 1519-1530.

Weber, I., Gerisch, G., Heizer, C., Murphy, J., Badelt, K., Stock, A., Schwartz, J. and Faix, J. (1999a). Cytokinesis mediated through the recruitment of cortexillins into the cleavage furrow. EMBO J. 18, 586-594

Weber, I., Niewöhner, J. and Faix, J. (1999b). Cytoskeletal protein mutations and cell motility in Dictyostelium. Biochem. Soc. Symp. 65, 245-265.

Zhang, J., Campbell, R. E., Ting, A. Y. and Tsien, R. Y. (2002). Creating new fluorescent probes for cell biology. Nat. Rev. Mol. Cell Biol. 3, 906-918. 